\title{
Research Article \\ On Invariant Tori of Nearly Integrable Hamiltonian Systems with Quasiperiodic Perturbation
}

\author{
Dongfeng Zhang ${ }^{1}$ and Rong Cheng ${ }^{2}$ \\ ${ }^{1}$ Department of Mathematics, Southeast University, Nanjing 210096, China \\ ${ }^{2}$ College of Mathematics and Physics, Nanjing University of Information Science and Technology, \\ Nanjing 210044, China \\ Correspondence should be addressed to Dongfeng Zhang, zhdf@seu.edu.cn
}

Received 2 September 2010; Accepted 25 October 2010

Academic Editor: Marlène Frigon

Copyright $@ 2010$ D. Zhang and R. Cheng. This is an open access article distributed under the Creative Commons Attribution License, which permits unrestricted use, distribution, and reproduction in any medium, provided the original work is properly cited.

We are concerned with the persistence of frequency of invariant tori for analytic integrable Hamiltonian system with quasiperiodic perturbation. It is proved that if the unperturbed system satisfies the Rüssmann's nondegeneracy condition and has nonzero Brouwer's topological degree at some Diophantine frequency; the perturbed system satisfies the colinked nonresonant condition, then the invariant torus with this frequency persists under quasiperiodic perturbation.

\section{Introduction and Main Results}

It is well known that the classical KAM theorem concludes that most of invariant tori of integrable Hamiltonian system can survive small perturbation under Kolmogorov's nondegeneracy condition [1-4]. What is more, the frequency of the persisting invariant tori remains the same. Later important generalizations of the classical KAM theorem were made to the Rüssmann's nondegeneracy condition [5-9]. However, in the case of Rüssmann's nondegeneracy condition, we can only get the existence of a family of invariant tori while there is no information on the persistence of frequency of any torus. Recently, Chow et al. [10] and Sevryuk [11] consider perturbations of moderately degenerate integrable Hamiltonian system and prove that the first $d$ frequencies $(d<n, n$ denotes the freedom of Hamiltonian system) of unperturbed invariant $n$-tori can persist. Xu and You [12] prove that if some frequency satisfies certain nonresonant condition and topological degree condition, the perturbed system still has an invariant torus with this frequency under Rüssmann's nondegeneracy condition. In this paper, we consider the case of quasiperiodic perturbation under Rüssmann's nondegeneracy condition. 
Consider the following Hamiltonian:

$$
H=h(y)+p(x, y, \tilde{\omega} t)
$$

where $y \in D \subset \mathbb{R}^{n}, x \in \mathbb{T}^{n}, \tilde{\omega} \in \mathbb{R}^{m}, h$ and $p$ are real analytic on a complex neighborhood of $D \times \mathbb{T}^{n} \times \mathbb{T}^{m}, D$ is a closed bounded domain, $\mathbb{T}^{n}, \mathbb{T}^{m}$ denote $n$-torus and $m$-torus, respectively, and $p(x, y, \tilde{\omega} t)$ is a perturbation and quasiperiodic in $\phi=\tilde{\omega} t$. Here, a function $f(t)$ is called a quasiperiodic function with the vector of basic frequencies $\tilde{\omega}=\left(\tilde{\omega}_{1}, \tilde{\omega}_{2}, \ldots, \tilde{\omega}_{m}\right)$ if there is function $f(t)=F\left(\phi_{1}, \phi_{2}, \ldots, \phi_{m}\right)$, where $F$ is $2 \pi$ periodic in all of its arguments $\phi_{j}=\tilde{\omega}_{j} t$ for $j=1,2, \ldots, m$.

After introducing two conjugate variables $\phi \bmod 2 \pi$ and $\eta$, the Hamiltonian (1.1) can be written in the form of an autonomous Hamiltonian with $n+m$ degrees of freedom as follows:

$$
H=h(y)+\langle\tilde{\omega}, \eta\rangle+p(x, y, \phi)
$$

Thus, the perturbed motion of Hamiltonian (1.1) is described by the following equations:

$$
\begin{gathered}
\dot{x}=H_{y}=h_{y}(y)+p_{y}(x, y, \phi), \\
\dot{y}=-H_{x}=-p_{x}(x, y, \phi), \\
\dot{\phi}=H_{\eta}=\tilde{\omega}, \\
\dot{\eta}=-H_{\phi}=-p_{\phi}(x, y, \phi) .
\end{gathered}
$$

Suppose that the frequency mapping $\bar{\omega}(y)=\partial h(y) / \partial y$ satisfies Rüssmann's nondegeneracy condition

$$
a_{1} \bar{\omega}_{1}(y)+a_{2} \bar{\omega}_{2}(y)+\cdots+a_{n} \bar{\omega}_{n}(y) \not \equiv 0 \quad \text { on } D
$$

for all $\left(a_{1}, a_{2}, \ldots, a_{n}\right) \in \mathbb{R}^{n} \backslash\{0\}$. The condition (1.4) is first given in [6] by Rüssmann, and it is the sharpest one for KAM theorems.

When $p=0$, the unperturbed system (1.3) has invariant tori $\mathbb{T}_{0}=\mathbb{T}^{n} \times \mathbb{T}^{m} \times\{0\} \times\{0\}$ with frequency $\omega=(\bar{\omega}(y), \tilde{\omega})$, carrying a quasiperiodic flow $x(t)=\bar{\omega}(y) t+x_{0}, \phi(t)=\tilde{\omega} t+\phi_{0}$.

When $p \neq 0$, given a frequency $\omega=\left(\bar{\omega}_{0}, \tilde{\omega}\right)$ satisfying certain Diophantine condition, we are concerned with the existence of invariant torus with $\omega$ as its frequency for Hamiltonian system (1.3). The following theorem will give a positive answer.

Theorem 1.1. Consider the real analytic Hamiltonian system (1.3). Let $\bar{\omega}(y)=h_{y}(y)$ and $\bar{\omega}_{0}=$ $\bar{\omega}\left(y_{0}\right), y_{0} \in D$. Suppose that $\omega=\left(\bar{\omega}_{0}, \tilde{\omega}\right)$ satisfies the Diophantine condition as follows:

$$
\left|\left\langle\bar{k}, \bar{\omega}_{0}\right\rangle+\langle\tilde{k}, \tilde{\omega}\rangle\right| \geq \frac{\alpha}{|k|^{\tau}}, \quad \forall 0 \neq k=(\bar{k}, \tilde{k}) \in Z^{n+m}
$$


and the Brouwer's topological degree of the frequency mapping $\bar{\omega}(y)$ at $\bar{\omega}_{0}$ on $D$ is not zero, that is,

$$
\operatorname{deg}\left(\bar{\omega}(y), D, \bar{\omega}_{0}\right) \neq 0
$$

then there exists a sufficiently small $\epsilon>0$, such that if

$$
\|p\|=\sup _{D \times \mathbb{T}^{n} \times \mathbb{T}^{m}}|p(x, y, \phi)| \leq \epsilon,
$$

the system (1.3) has an invariant torus with $\omega=\left(\bar{\omega}_{0}, \tilde{\omega}\right)$ as its frequency.

Remark 1.2. In [13] the authors only obtained the existence of invariant tori for Hamiltonian systems (1.3), while the frequency of the persisting invariant tori may have some drifts.

As in [4], instead of proving Theorem 1.1 directly, we are going to deduce it from another KAM theorem, which is concerned with perturbations of a family of linear Hamiltonians. This is accomplished by introducing a parameter and changing the Hamiltonian system (1.3) to a parameterized system. For $\xi \in D$, let $y=\xi+z$, then

$$
H=e(\xi)+\langle\bar{\omega}(\xi), z\rangle+\langle\tilde{\omega}, \eta\rangle+p(x, \xi+z, \phi)+O\left(\left|z^{2}\right|\right)
$$

where $e(\xi)=h(\xi), \bar{\omega}(\xi)=h_{y}(\xi), \xi \in D$ is regarded as parameters. Since $e(\xi)$ is an energy constant, which is usually omitted, and the term $O\left(\left|z^{2}\right|\right)$ can be taken as a new perturbation, we consider the Hamiltonian

$$
\begin{aligned}
H(x, z, \phi, \eta ; \xi) & =\langle\bar{\omega}(\xi), z\rangle+\langle\tilde{\omega}, \eta\rangle+P(x, z, \phi ; \xi) \\
& =N+P
\end{aligned}
$$

where $N=\langle\bar{\omega}(\xi), z\rangle+\langle\tilde{\omega}, \eta\rangle$ is a normal form, $P=P(x, z, \phi ; \xi)$ is a small perturbation.

Let

$$
\begin{aligned}
D(s, r) & =\{(x, \phi, z, \eta)|| \operatorname{Im} x|\leq s,| \operatorname{Im} \phi|\leq s,| z|\leq r,| \eta \mid \leq r\} \\
& \subset \mathbb{C}^{n} / 2 \pi \mathbb{Z}^{n} \times \mathbb{C}^{m} / 2 \pi \mathbb{Z}^{m} \times \mathbb{C}^{n} \times \mathbb{C}^{m}, \\
\Lambda & =\{\xi \in D \mid \operatorname{dist}(\xi, \partial D) \geq \sigma\},
\end{aligned}
$$

where $\sigma \geq r>0$ is a small constant. Let $\Lambda_{\sigma}$ be the complex neighborhood of $\Lambda$ with the radius $\sigma$, that is,

$$
\Lambda_{\sigma}=\left\{\xi \in \mathbb{C}^{n} \mid \operatorname{dist}(\xi, \Lambda) \leq \sigma\right\}
$$


Now, the Hamiltonian $H(x, \phi, z, \eta ; \xi)$ is real analytic on $D(s, r) \times \Lambda_{\sigma}$. The corresponding Hamiltonian system becomes

$$
\begin{gathered}
\dot{x}=H_{z}=\bar{\omega}(\xi)+P_{z}(x, z, \phi ; \xi), \\
\dot{z}=-H_{x}=-P_{x}(x, z, \phi ; \xi), \\
\dot{\phi}=H_{\eta}=\tilde{\omega}, \\
\dot{\eta}=-H_{\phi}=-P_{\phi}(x, z, \phi ; \xi) .
\end{gathered}
$$

Thus, the persistence of invariant tori for nearly integrable Hamiltonian system (1.3) is reduced to the persistence of invariant tori for the family of Hamiltonian system (1.12) depending on the parameter $\xi$.

We expand

$$
P(x, z, \phi ; \xi)=\sum_{(\bar{k}, \tilde{k}) \in \mathbb{Z}^{n} \times \mathbb{Z}^{m}} P_{k}(z ; \xi) e^{i(\langle\bar{k}, x\rangle+\langle\tilde{k}, \phi\rangle)},
$$

then we define

$$
\|P\|_{D(s, r) \times \Lambda_{\sigma}}=\sup _{D(s, r) \times \Lambda_{\sigma}}\left|\sum_{(\bar{k}, \tilde{k}) \in \mathbb{Z}^{n} \times \mathbb{Z}^{m}} P_{k}(z ; \xi) e^{i(\langle\bar{k}, x\rangle+\langle\tilde{k}, \phi\rangle)}\right| .
$$

Theorem 1.3. Suppose that $H(x, z, \phi, \eta ; \xi)=\langle\bar{\omega}(\xi), z\rangle+\langle\tilde{\omega}, \eta\rangle+P(x, z, \phi ; \xi)$ is real analytic on $D(s, r) \times \Lambda_{\sigma}$. Let $\bar{\omega}_{0}=\bar{\omega}\left(\xi_{0}\right), \xi_{0} \in \Lambda$. Suppose that $\bar{\omega}_{0}$ satisfies (1.5) and $\operatorname{deg}\left(\bar{\omega}(\xi), \Lambda, \bar{\omega}_{0}\right) \neq 0$, then there exists a sufficiently small $\epsilon>0$, such that if $\|P\|_{D(s, r) \times \Lambda_{\sigma}} \leq \epsilon$, there exists $\xi_{*} \in \Lambda$, such that the Hamiltonian system (1.12) at $\xi=\xi_{*}$ has an invariant torus with $\left(\bar{\omega}_{0}, \tilde{\omega}\right)$ as its frequency.

\section{Proof of the Main Results}

In order to prove Theorem 1.3, we introduce an external parameter $\lambda$ and consider the following Hamiltonian system:

$$
\begin{gathered}
\dot{x}=H_{z}=\bar{\omega}(\xi)+\lambda+P_{z}(x, z, \phi ; \xi), \\
\dot{z}=-H_{x}=-P_{x}(x, z, \phi ; \xi), \\
\dot{\phi}=H_{\eta}=\tilde{\omega}, \\
\dot{\eta}=-H_{\phi}=-P_{\phi}(x, z, \phi ; \xi),
\end{gathered}
$$

where $H(x, z, \phi, \eta ; \xi, \lambda)=\langle\bar{\omega}(\xi)+\lambda, z\rangle+\langle\tilde{\omega}, \eta\rangle+P(x, z, \phi ; \xi)$. When $\lambda=0$, the Hamiltonian system (2.1) comes back to the system (1.12). The idea of introducing outer parameters was used in $[8,11,12]$. We first give a KAM theorem for Hamiltonian system with parameters $(\xi, \lambda)$. 
Fixed Point Theory and Applications

Let $d=\max _{\xi, \gamma \in \Lambda_{\sigma}}|\bar{\omega}(\xi)-\bar{\omega}(\gamma)|$ and define

$$
B(\bar{\omega}(\xi), d)=\left\{\lambda \in \mathbb{C}^{n} \mid \operatorname{dist}(\lambda, \bar{\omega}(\xi))<d\right\}
$$

Let $\mathcal{O}=\left(\bigcup_{\xi \in \Lambda_{\sigma}} B(\bar{\omega}(\xi), d)\right) \cap \mathbb{R}^{n}$. We have $\bar{\omega}(\Lambda)=\{\bar{\omega}(\xi) \mid \xi \in \Lambda\} \subset \mathcal{O}$, and define

$$
\mathcal{O}_{\alpha}=\left\{\Omega \in \mathcal{O}||\langle\bar{k}, \Omega\rangle+\langle\tilde{k}, \tilde{\omega}\rangle \mid \geq \frac{\alpha}{|k|^{\tau}}, \forall 0 \neq k=(\bar{k}, \tilde{k}) \in \mathbb{Z}^{n+m}\right\} .
$$

Let $K>0$ and $h=\alpha / 2 K^{\tau+1}$. Denote $\mathcal{O}_{\alpha, h}$ the complex neighborhood of $\mathcal{O}_{\alpha}$ with radius $h$, then for any $\Omega \in \mathcal{O}_{\alpha, h}$, we have

$$
|\langle\bar{k}, \Omega\rangle+\langle\tilde{k}, \tilde{\omega}\rangle| \geq \frac{\alpha}{2|k|^{\tau}}, \quad \forall 0 \neq|k| \leq K .
$$

Let $\Pi=\Lambda_{\sigma} \times B(0,2 d+1)$. The Hamiltonian $H(x, z, \phi, \eta ; \xi, \lambda)$ is real analytic on $D(s, r) \times \Pi$.

Theorem 2.1. Consider the parameterized Hamiltonian system (2.1), which is real analytic on $D(s, r) \times \Pi$. Then there exists a sufficiently small $\epsilon>0$, such that if $\|P\|_{D(s, r) \times \Pi} \leq \epsilon$, there exists a Cantor-like family of analytic curves

$$
\Gamma_{\Omega}^{*}=\left\{(\xi, \lambda(\xi)) \mid \xi \in \Lambda, \Omega \in \mathcal{O}_{\alpha}\right\} \subset \Pi,
$$

which are determined implicitly by the equation

$$
\lambda+\bar{\omega}(\xi)+F_{*}(\xi, \lambda)=\Omega,
$$

where $F_{*}(\xi, \lambda)$ is $C^{\infty}$-smooth in $(\xi, \lambda)$ on $\Pi$ and satisfies

$$
\left|F_{*}(\xi, \lambda)\right| \leq \frac{2 \epsilon}{r}, \quad\left|F_{* \xi}(\xi, \lambda)\right|+\left|F_{* \lambda}(\xi, \lambda)\right| \leq \frac{1}{2},
$$

and a parameterized family of symplectic mappings

$$
\Psi_{*}(\cdot, \cdot ; \xi, \lambda): D\left(\frac{s}{2}, \frac{r}{2}\right) \longrightarrow D(s, r), \quad(\xi, \lambda) \in \Gamma^{*}=\bigcup_{\Omega \in \mathcal{O}_{\alpha}} \Gamma_{\Omega^{\prime}}^{*}
$$

where $\Psi_{*}$ is $C^{\infty}$-smooth in $(\xi, \lambda)$ on $\Gamma^{*}$ in the sense of Whitney and analytic in $(x, \phi, z)$ on $D(s / 2, r / 2)$, such that for each $(\xi, \eta) \in \Gamma^{*}$, one has

$$
H \circ \Psi_{*}=\langle\Omega, z\rangle+\langle\tilde{\omega}, \eta\rangle+P_{*}(x, z, \phi ; \xi, \lambda),
$$

where $P_{*}(x, z, \phi ; \xi, \lambda)=O\left(|z|^{2}\right)$ near $z=0$. Thus, the perturbed system (2.1) possesses invariant tori with $(\Omega, \tilde{\omega})$ as its frequency. 
Remark 2.2. The derivatives in the estimates of (2.7) should be understood in the sense of Whitney [14]. In fact, we can extend $F_{*}(\xi, \lambda)$ to a neighborhood of $\Gamma_{\Omega}^{*}$ as a consequence in [15].

Remark 2.3. In fact, we can prove that $\Psi_{*}$ is Gevrey smooth with respect to the parameters $(\xi, \lambda)$ in the sense of Whitney as in [16-18].

Proof of Theorem 1.3. Now, we use the results of Theorem 2.1 to prove Theorem 1.3. In fact, Let $\Omega=\bar{\omega}_{0}$, then we have an analytic curve $\Gamma_{\bar{\omega}_{0}}^{*}: \lambda=\lambda(\xi), \xi \in \Lambda$, which is determined by the equation $\lambda+\bar{\omega}(\xi)+F_{*}(\xi, \lambda)=\bar{\omega}_{0}$. By implicit function theorem, we have

$$
\lambda(\xi)=\bar{\omega}_{0}-\bar{\omega}(\xi)+\lambda_{*}(\xi), \quad \xi \in \Lambda,
$$

where $\lambda_{*}(\xi)$ satisfies that

$$
\left|\lambda_{*}(\xi)\right| \leq \frac{2 \epsilon}{r}, \quad\left|\lambda_{* \xi}(\xi)\right| \leq \frac{4 \epsilon}{r}
$$

By the assumption $\operatorname{deg}\left(\bar{\omega}(\xi), \Lambda, \bar{\omega}_{0}\right) \neq 0$, if $\epsilon$ is sufficiently small, we have

$$
\operatorname{deg}(\lambda(\xi), \Lambda, 0)=\operatorname{deg}\left(\bar{\omega}_{0}-\bar{\omega}(\xi), \Lambda, 0\right) \neq 0
$$

Therefore, we have some $\xi_{*} \in \Lambda$ such that $\lambda\left(\xi_{*}\right)=0$. When $\lambda\left(\xi_{*}\right)=0$, the Hamiltonian system (2.1) comes back to the system (1.12). Therefore, by Theorem 2.1, at $\xi_{*}$ the Hamiltonian system (1.12) has an invariant torus with $\left(\bar{\omega}_{0}, \tilde{\omega}\right)$ as its frequency.

Now, it remains to prove Theorem 2.1. Our method is the standard KAM iteration. The difficulty is how to deal with parameters in KAM iteration.

\section{KAM Step}

The KAM step can be summarized in the following lemma.

Lemma 2.4. Consider real analytic Hamiltonian

$$
H=\langle\Omega(\xi, \lambda), z\rangle+\langle\tilde{\omega}, \eta\rangle+P(x, z, \phi ; \xi, \lambda),
$$

which is defined on $D(s, r) \times \Pi$, where $\Omega(\xi, \lambda)=\bar{\omega}(\xi)+\lambda+f(\xi, \lambda)$. Suppose that

$$
\|P\|_{D(s, r) \times \Pi} \leq \epsilon .
$$

Suppose that the function $f(\xi, \lambda)$ satisfies that

$$
\left|f_{\xi}(\xi, \lambda)\right|+\left|f_{\lambda}(\xi, \lambda)\right|<\frac{1}{2}, \quad \forall(\xi, \lambda) \in \Pi
$$


Fixed Point Theory and Applications

and then for all $\Omega \in \mathcal{O}_{\alpha}$, the equation

$$
\Omega(\xi, \lambda)=\bar{\omega}(\xi)+\lambda+f(\xi, \lambda)=\Omega
$$

defines implicitly an analytic mapping as follows:

$$
\lambda: \xi \in \Lambda_{\sigma} \longrightarrow \lambda(\xi) \in B(0,2 d+1)
$$

such that $\Gamma_{\Omega}=\left\{(\xi, \lambda(\xi)) \mid \xi \in \Lambda_{\sigma}\right\} \subset \Pi$. Moreover one defines

$$
\begin{gathered}
\delta=\frac{\alpha}{2 L K^{\tau+1}} \quad \text { with } L=2+\max _{\xi \in \Lambda_{\sigma}}\left|\bar{\omega}_{\xi}(\xi)\right|, \\
B\left(\Gamma_{\Omega}, \delta\right)=\left\{\left(\xi, \lambda^{\prime}\right) \in \mathbb{C}^{n} \times \mathbb{C}^{n},(\xi, \lambda) \in \Gamma_{\Omega}|| \lambda^{\prime}-\lambda \mid \leq \delta\right\} \subset \Pi .
\end{gathered}
$$

Then there exist $\Pi_{+} \subset \Pi$ and $D\left(s_{+}, r_{+}\right)$, such that for any $(\xi, \lambda) \in \Pi_{+}$there exists a symplectic mapping

$$
\Phi(\cdot, \cdot ; \xi, \lambda): D\left(s_{+}, r_{+}\right) \longrightarrow D(s, r)
$$

such that

$$
H_{+}=H \circ \Phi=\left\langle\Omega_{+}(\xi, \lambda), z\right\rangle+\langle\tilde{\omega}, \eta\rangle+P_{+}(x, z, \phi ; \xi, \lambda)
$$

where $\Omega_{+}(\xi, \lambda)=\bar{\omega}(\xi)+\lambda+f(\xi, \lambda)+f_{+}(\xi, \lambda)$. Moreover, the new perturbation satisfies

$$
\left\|P_{+}\right\|_{D\left(s_{+}, r_{+}\right) \times \Pi_{+}} \leq c\left[\frac{\epsilon^{2}}{\alpha r \rho^{n+\tau+1}}+\left(K^{n} e^{-K \rho}+\mu^{2}\right) \epsilon\right],
$$

where $s_{+}=s-5 \rho, r_{+}=\mu r$, and

$$
\Pi_{+}=\left\{\left(\xi, \lambda^{\prime}\right) \in \mathbb{C}^{n} \times \mathbb{C}^{n}\left|\xi \in \Lambda_{\sigma-(1 / 2) \delta},(\xi, \lambda) \in \Gamma,\right| \lambda^{\prime}-\lambda \mid \leq \frac{1}{2} \delta\right\},
$$

with $\Gamma=\bigcup_{\Omega \in O_{\alpha}} \Gamma_{\Omega}$.

The term $f_{+}(\xi, \lambda)$ which may generate the drift of frequency after one KAM step satisfies that

$$
\begin{gathered}
\left|f_{+}(\xi, \lambda)\right| \leq \frac{\epsilon}{r}, \quad \forall(\xi, \lambda) \in \Pi, \\
\left|f_{+\xi}(\xi, \lambda)\right|+\left|f_{+\lambda}(\xi, \lambda)\right| \leq \frac{2 \epsilon}{\delta r}, \quad \forall(\xi, \lambda) \in \Pi_{+} .
\end{gathered}
$$

Thus, if

$$
\frac{2 \epsilon}{\delta r} \leq \frac{1}{4}
$$


then the equation

$$
\Omega_{+}(\xi, \lambda)=\bar{\omega}(\xi)+\lambda+f(\xi, \lambda)+f_{+}(\xi, \lambda)=\Omega
$$

determines an analytic mapping

$$
\lambda_{+}: \xi \in \Lambda_{\sigma_{+}} \longrightarrow \lambda_{+}(\xi) \in B(0,2 d+1)
$$

with $\sigma_{+}=\sigma-(1 / 2) \delta$, satisfying

$$
\begin{gathered}
\left|\lambda_{+}(\xi)-\lambda(\xi)\right| \leq \frac{2 \epsilon}{r} \leq \frac{\delta}{4}, \\
\Gamma_{\Omega}^{+}=\left\{\left(\xi, \lambda_{+}(\xi)\right) \mid \xi \in \Lambda_{\sigma_{+}}\right\} \subset \Pi_{+} .
\end{gathered}
$$

For $K_{+}>0$, define $\delta_{+}=\alpha / 2 L K_{+}^{\tau+1}$. If

$$
\delta_{+}<\frac{\delta}{4}
$$

then for all $\Omega \in \mathcal{O}_{\alpha}$ one has $B\left(\Gamma_{\Omega^{\prime}}^{+}, \delta_{+}\right) \subset \Pi_{+}$.

Proof of Lemma 2.4. We divide the proof into several parts.

\section{(A) Truncation}

Since $P$ is real analytic, consider the Taylor-Fourier series of $P$ as follows:

$$
P=\sum_{\bar{k} \in Z^{n}, \tilde{k} \in Z^{m}, q \in Z^{n}} P_{\widetilde{k} \tilde{k} q}(\xi, \lambda) z^{q} e^{i(\langle\bar{k}, x\rangle+\langle\tilde{k}, \phi\rangle)} .
$$

Let the truncation $R$ of $P$ have the following form:

$$
R=\sum_{\bar{k} \in Z^{n}, \tilde{k} \in Z^{m},|k| \leq K}\left(P_{\bar{k} \tilde{k} 0}+\left\langle P_{\bar{k} \tilde{k} 1}, z\right\rangle\right) e^{i(\langle\bar{k}, x\rangle+\langle\tilde{k}, \phi\rangle)},
$$

where $|k|=|\bar{k}|+|\tilde{k}|, K$ is a positive constant. Then,

$$
\|R\|_{D(s-\rho, r) \times \Pi} \leq c \epsilon, \quad\|P-R\|_{D(s-\rho, 2 \mu r) \times \Pi} \leq c\left(K^{n} e^{-K \rho} \epsilon+\mu^{2} \epsilon\right) .
$$


(B) Extending the Small Divisor Estimate

By (2.16), the Diophantine condition (2.3) is satisfied for $\langle\bar{k}, \Omega(\xi, \lambda)\rangle+\langle\tilde{k}, \tilde{\omega}\rangle$, that is, for all parameters $(\xi, \lambda) \in \Gamma=\bigcup_{\Omega \in \mathcal{O}_{\alpha}} \Gamma_{\Omega}$. Moreover, the definition (2.18) of $\delta$ implies that

$$
|\langle\bar{k}, \Omega(\xi, \lambda)\rangle+\langle\tilde{k}, \tilde{\omega}\rangle| \geq \frac{\alpha}{2|k|^{\tau}}, \quad \forall 0 \neq|k| \leq K,
$$

for all $(\xi, \lambda) \in B(\Gamma, \delta)$. Indeed, for all $\left(\xi^{\prime}, \lambda^{\prime}\right) \in B(\Gamma, \delta)$, there is some $(\xi, \lambda) \in \Gamma$ satisfying $\left|\xi-\xi^{\prime}\right|+\left|\lambda-\lambda^{\prime}\right| \leq \delta$, hence

$$
\begin{aligned}
\left|\left\langle\bar{k}, \Omega\left(\xi^{\prime}, \lambda^{\prime}\right)-\Omega(\xi, \lambda)\right\rangle\right| & =\left|\left\langle\bar{k}, \bar{\omega}\left(\xi^{\prime}\right)-\bar{\omega}(\xi)+\lambda^{\prime}-\lambda+f\left(\xi^{\prime}, \lambda^{\prime}\right)-f(\xi, \lambda)\right\rangle\right| \\
& \leq|\bar{k}|\left[\left(\left|\bar{\omega}_{\xi}\right|+\left|f_{\xi}\right|\right)\left|\xi^{\prime}-\xi\right|+\left(1+\left|f_{\lambda}\right|\right)\left|\lambda^{\prime}-\lambda\right|\right] \\
& \leq|\bar{k}|\left(2+\left|\bar{\omega}_{\xi}\right|\right) \frac{\alpha}{2 L K^{\tau+1}} \leq \frac{\alpha}{2|k|^{\tau}}
\end{aligned}
$$

for $0<|\bar{k}|+|\tilde{k}| \leq K$. Together with the estimate (2.3) for $\langle\bar{k}, \Omega(\xi, \lambda)\rangle+\langle\tilde{k}, \tilde{\omega}\rangle$, this proves the claim.

\section{(C) Construction of the Symplectic Mapping}

The aim of this section is to find a Hamiltonian $F$, such that the time 1-map $\Phi=\left.X_{F}^{t}\right|_{t=1}$ carries $H$ into a new normal form with a smaller perturbation. Formally, we assume that $F$ is of the following form:

$$
F=\sum_{0 \neq|k| \leq K}\left(F_{\bar{k} \tilde{k} 0}+\left\langle F_{\bar{k} \tilde{k} 1}, z\right\rangle\right) e^{i(\langle\bar{k}, x\rangle+\langle\tilde{k}, \phi\rangle)}
$$

if

$$
\{N, F\}=[R]-R,
$$

where $\{\cdot, \cdot\}$ is the Poisson bracket, $[R]=\int_{\mathbb{T}^{m}}\left[\int_{\mathbb{T}^{n}} R d x\right] d \phi$, then,

$$
\begin{aligned}
H \circ \Phi= & (N+R) \circ \Phi+(P-R) \circ \Phi \\
= & N+[R]+\{N, F\}+R-[R] \\
& +\int_{0}^{1}\{(1-t)\{N, F\}+R, F\} \circ X_{F}^{t} d t+(P-R) \circ \Phi \\
= & N_{+}+P_{+},
\end{aligned}
$$

where $N_{+}=N+[R]=\left\langle\Omega_{+}(\xi, \lambda), z\right\rangle+\langle\tilde{\omega}, \eta\rangle, P_{+}=\int_{0}^{1}\{(1-t)\{N, F\}+R, F\} \circ X_{F}^{t} d t+(P-R) \circ \Phi$. 
Putting (2.32) and (2.36) into (2.37) yields

$$
\begin{aligned}
& \sum_{0 \neq|k| \leq K} i(\langle\bar{k}, \Omega(\xi, \lambda)\rangle+\langle\tilde{k}, \tilde{\omega}\rangle)\left(F_{\bar{k} \tilde{k} 0}+\left\langle F_{\bar{k} \tilde{k} 1}, z\right\rangle\right) e^{i(\langle\bar{k}, x\rangle+\langle\tilde{k}, \phi\rangle)} \\
& =\sum_{0 \neq|k| \leq K}\left(P_{\bar{k} \tilde{k} 0}+\left\langle P_{\bar{k} \tilde{k} 1}, z\right\rangle\right) e^{i(\langle\bar{k}, x\rangle+\langle\tilde{k}, \phi\rangle)} .
\end{aligned}
$$

Equation (2.39) is solvable because the Diophantine condition (2.34) is satisfied for all parameters $(\xi, \lambda) \in B(\Gamma, \delta)$, then we have

$$
F=\sum_{0 \neq|k| \leq K} \frac{\left(P_{\bar{k} \tilde{k} 0}+\left\langle P_{\bar{k} \tilde{k} 1}, z\right\rangle\right) e^{i(\langle\bar{k}, x\rangle+\langle\tilde{k}, \phi\rangle)}}{i(\langle\bar{k}, \Omega(\xi, \lambda)\rangle+\langle\tilde{k}, \tilde{\omega}\rangle)}, \quad \forall(\xi, \lambda) \in B(\Gamma, \delta),
$$

which satisfies $\|F\|_{D(s-2 \rho, r) \times B(\Gamma, \delta)} \leq c \epsilon / \alpha \rho^{\tau+n}$.

Moreover, with the estimate of Cauchy, we get $\left\|F_{x}\right\|_{D(s-3 \rho, r)} \leq c \epsilon / \alpha \rho^{\tau+n+1}$, $\left\|F_{\phi}\right\|_{D(s-3 \rho, r)} \leq c \epsilon / \alpha \rho^{\tau+n+1}$, and $\left\|F_{z}\right\|_{D(s-2 \rho, r / 2)} \leq c \epsilon / \alpha r \rho^{\tau+n}$, hence

$$
\frac{1}{r}\left\|F_{x}\right\|, \quad \frac{1}{r}\left\|F_{\phi}\right\|, \quad \frac{1}{\rho}\left\|F_{z}\right\| \leq \frac{c \epsilon}{\alpha r \rho^{\tau+n+1}},
$$

uniformly on $D(s-3 \rho, r / 2) \times B(\Gamma, \delta)$.

\section{(D) Estimates of the Symplectic Mapping}

The coordinate transformation $\Phi$ is obtained as the time 1-map of the flow $X_{F}^{t}$ of the Hamiltonian vectorfield $X_{F}$, with equations

$$
\dot{z}=-F_{x}, \quad \dot{\eta}=-F_{\phi}, \quad \dot{x}=F_{z}, \quad \dot{\phi}=F_{\eta}=0 .
$$

Thus, if $0<\mu \leq 1 / 8$ and $\epsilon$ is sufficiently small, we have for all $(\xi, \lambda) \in B(\Gamma, \delta)$,

$$
\begin{gathered}
\Phi(\cdot, \cdot ; \xi, \lambda)=X_{F}^{1}:(s-4 \rho, 2 \mu r) \longrightarrow(s-3 \rho, 3 \mu r) \\
\left|U_{1}-i d\right| \leq\left\|F_{x}\right\| \leq \frac{c \epsilon}{\alpha \rho^{\tau+n+1}}, \quad\left|U_{2}-i d\right| \leq\left\|F_{\phi}\right\| \leq \frac{c \epsilon}{\alpha \rho^{\tau+n+1}} \\
|V-i d| \leq\left\|F_{z}\right\| \leq \frac{c \epsilon}{\alpha r \rho^{\tau+n}}
\end{gathered}
$$

on $D(s-4 \rho, 2 \mu r) \times B(\Gamma, \delta)$ for $\Phi=\left(U_{1}(x, \phi, z), U_{2}(x, \phi, z), V(x, \phi)\right)$, where $U_{1}, U_{2}$ is affine in $z$, and $V$ is independent of $z$.

Let $W=\operatorname{diag}\left(r^{-1} I_{n}, r^{-1} I_{m}, \rho^{-1} I_{n}\right)$, where $I_{n}$ is the $n$th unit matrix. Thus, it follows that

$$
\|W(\Phi-i d)\|_{D(s-4 \rho, 2 \mu r) \times B(\Gamma, \delta)} \leq \frac{c \epsilon}{\alpha r \rho^{\tau+n+1}} .
$$


By the preceding estimates and the Cauchy's estimate, we have

$$
\left\|W(D \Phi-I d) W^{-1}\right\|_{D(s-5 \rho, \mu r) \times B(\Gamma, \delta)} \leq \frac{c \epsilon}{\alpha r \rho^{\tau+n+1}},
$$

where $D \Phi$ denotes the Jacobian matrix with respect to $(z, x, \phi)$.

\section{(E) Estimates of New Error Term}

To estimate $P_{+}$, we first consider the term $\{R, F\}$. By Cauchy's estimate,

$$
\begin{aligned}
\|\{R, F\}\|_{D(s-3 \rho, r / 2)} & \leq c\left(\left\|R_{z}\right\|\left\|F_{x}\right\|+\left\|R_{x}\right\|\left\|F_{z}\right\|\right) \\
& \leq c\left(\frac{\epsilon}{r} \cdot \frac{\epsilon}{\alpha \rho^{\tau+n+1}}+\frac{\epsilon}{\rho} \cdot \frac{\epsilon}{\alpha r \rho^{\tau+n}}\right) \leq \frac{c \epsilon^{2}}{\alpha r \rho^{\tau+n+1}} .
\end{aligned}
$$

The same holds for $\|\{\{N, F\}, F\}\|_{D(s-3 \rho, r / 2)}$. Together with (2.43) and $\mu \leq 1 / 8$, we get

$$
\begin{aligned}
& \left\|\int_{0}^{1}\{(1-t)\{N, F\}+R, F\} \circ X_{F}^{t} d t\right\|_{D(s-5 \rho, \mu r)} \\
& \quad \leq\|\{(1-t)\{N, F\}+R, F\}\|_{D(s-4 \rho, 2 \mu r)} \leq \frac{c \epsilon^{2}}{\alpha r \rho^{\tau+n+1}} .
\end{aligned}
$$

The other term in $P_{+}$is bounded by

$$
\begin{aligned}
\|(P-R) \circ \Phi\|_{D(s-5 \rho, \mu r)} & \leq\|(P-R)\|_{D(s-4 \rho, 2 \mu r)} \\
& \leq c\left(K^{n} e^{-K \rho} \epsilon+\mu^{2} \epsilon\right) .
\end{aligned}
$$

Let $s_{+}=s-5 \rho, r_{+}=\mu r$. The preceding estimates are uniform in the domain of parameters $B(\Gamma, \delta)$, so the new perturbation satisfies that

$$
\left\|P_{+}\right\|_{D\left(s_{+}, r_{+}\right) \times \Pi_{+}} \leq c\left[\frac{\epsilon^{2}}{\alpha r \rho^{\tau+n+1}}+\left(K^{n} e^{-K \rho}+\mu^{2}\right) \epsilon\right] .
$$

Since $f_{+}(\xi, \lambda)=P_{001}$, the estimate for $f_{+}$holds. Let $\Pi_{+}$be defined as in Lemma 2.4, we have $\operatorname{dist}\left(\Pi_{+}, \partial \Pi\right) \geq(1 / 2) \delta$. Then, for all $(\xi, \lambda) \in \Pi_{+}$, the Cauchy's estimate yields the estimate for $f_{+\xi}(\xi, \lambda)$ and $f_{+\lambda}(\xi, \lambda)$. Moreover, by $(2.25)$, we have

$$
\left|\frac{\partial \Omega_{+}(\xi, \lambda)}{\partial \lambda}\right| \geq 1-\left|f_{\lambda}(\xi, \lambda)\right|-\left|f_{+\lambda}(\xi, \lambda)\right| \geq \frac{1}{4} \neq 0 .
$$

Thus, by the implicit function theorem, the equation

$$
\Omega_{+}(\xi, \lambda)=\bar{\omega}(\xi)+\lambda+f(\xi, \lambda)+f_{+}(\xi, \lambda)=\Omega
$$


determines an analytic curve

$$
\lambda_{+}: \xi \in \Lambda_{\sigma_{+}} \longrightarrow \lambda_{+}(\xi) .
$$

Moreover, we have

$$
\begin{aligned}
\left|\lambda_{+}(\xi)-\lambda(\xi)\right| & \leq\left|f_{\lambda}\right| \cdot\left|\lambda_{+}-\lambda\right|+\left|f_{+}\left(\xi, \lambda_{+}\right)\right| \\
& \leq \frac{1}{2}\left|\lambda_{+}-\lambda\right|+\frac{\epsilon}{r}
\end{aligned}
$$

this proves (2.28). By the estimates (2.28) and (2.30), the conclusion $\Gamma_{\Omega}^{+} \subset \Pi_{+}, B\left(\Gamma_{\Omega^{\prime}}^{+}, \delta_{+}\right) \subset \Pi_{+}$ holds. Thus, the proof of Lemma 2.4 is complete.

\section{KAM Iteration}

In this section, we have two tasks which ensure that the above iteration can go on infinitely. The first one is to choose some suitable parameters, the other one is to verify some assumptions in Lemma 2.4 .

For given $\rho_{0}=s / 20, r_{0}=r, s_{0}=s, \epsilon_{0}=\alpha r_{0} \rho_{0}^{\tau+n+1} E_{0}$, and $\mu_{0}=E_{0}^{1 / 2}, K_{0}$ is determined by $K_{0}^{n} e^{-K_{0} \rho_{0}}=E_{0}$, we define $\rho_{j+1}=\rho_{j} / 2, s_{j+1}=s_{j}-5 \rho_{j}, \mu_{j}=E_{j}^{1 / 2}, r_{j+1}=\mu_{j} r_{j}, E_{j+1}=c E_{j}^{3 / 2}$, and $\epsilon_{j+1}=\alpha r_{j+1} \rho_{j+1}^{\tau+n+1} E_{j+1}, K_{j+1}$ is determined by the equation $K_{j+1}^{n} e^{-K_{j+1} \rho_{j+1}}=E_{j+1}$.

Let $\Pi_{0}=\Lambda_{0} \times B(0,2 d+1), D_{0}=D\left(s_{0}, r_{0}\right)$. By the iteration lemma, we have a sequence of parameter sets $\Pi_{j}$ with $\Pi_{j+1} \subset \Pi_{j}$ and a sequence of symplectic mappings $\Phi_{j}$ such that $\Phi_{j}: D_{j+1} \times \Pi_{j+1} \rightarrow D_{j} \times \Pi_{j}$, where $D_{j}=D\left(s_{j}, r_{j}\right)$. Moreover, we have

$$
\begin{gathered}
\left\|W_{j}\left(\Phi_{j}-i d\right)\right\|_{D_{j} \times \Pi_{j}} \leq c E_{j}, \\
\left\|W_{j}\left(D \Phi_{j}-I d\right) W_{j}^{-1}\right\|_{D_{j} \times \Pi_{j}} \leq c E_{j},
\end{gathered}
$$

where $W_{j}=\operatorname{diag}\left(r_{j}^{-1} I_{n}, r_{j}^{-1} I_{m}, \rho_{j}^{-1} I_{n}\right)$.

Let $\Psi_{j}=\Phi_{0} \circ \Phi_{1} \circ \cdots \circ \Phi_{j-1}$ with $\Psi_{0}=i d$, then

$$
H_{j}=H_{0} \circ \Psi_{j}=N_{j}+P_{j}
$$

where $N_{j}=\left\langle\Omega_{j}(\xi, \lambda), z\right\rangle+\langle\tilde{\omega}, \eta\rangle$, and $\Omega_{j}(\xi, \lambda)=\bar{\omega}(\xi)+\lambda+\Sigma_{i=0}^{j-1} f_{i}(\xi, \lambda)$.

Let $\delta_{j}=\alpha / 2 L K_{j}^{\tau+1}, \sigma_{j}=\sigma_{j-1}-(1 / 2) \delta_{j-1}$, where $L=2+\max _{\xi \in \Lambda_{\sigma_{j}}}\left|\bar{\omega}_{\xi}(\xi)\right|, \sigma_{0}=\sigma$. From the iteration lemma, we have that for all $\Omega \in \mathcal{O}_{\alpha}$, the equation

$$
\Omega_{j}(\xi, \lambda)=\bar{\omega}(\xi)+\lambda+\Sigma_{i=0}^{j-1} f_{i}(\xi, \lambda)=\Omega
$$


on $\Pi_{j}$ defines implicitly an analytic mapping $\lambda=\lambda_{j}(\xi), \xi \in \Lambda_{\sigma_{j}}$, whose image in $\Pi_{j}$ forms an analytic curve $\Gamma_{\Omega}^{j}$. Let $\Gamma^{j}=\bigcup_{\Omega \in \mathcal{O}_{\alpha}} \Gamma_{\Omega}^{j}$. We define

$$
\Pi_{j+1}=\left\{\left(\xi, \lambda^{\prime}\right) \in \mathbb{C}^{n} \times \mathbb{C}^{n}\left|\xi \in \Lambda_{\sigma_{j+1}},(\xi, \lambda) \in \Gamma^{j},\right| \lambda^{\prime}-\lambda \mid \leq \frac{1}{2} \delta_{j}\right\},
$$

which satisfies the property $\Pi_{j+1} \subset \Pi_{j}, \operatorname{dist}\left(\Pi_{j+1}, \partial \Pi_{j}\right) \geq(1 / 2) \delta_{j}$.

Let $f_{j}(\xi, \lambda)=\Omega_{j+1}(\xi, \lambda)-\Omega_{j}(\xi, \lambda)$, then we have

$$
\begin{gathered}
\left|f_{j}(\xi, \lambda)\right| \leq \frac{\epsilon_{j}}{r_{j}}, \quad \forall(\xi, \lambda) \in \Pi_{j}, \\
\left|f_{j \xi}(\xi, \lambda)\right|+\left|f_{j \lambda}(\xi, \lambda)\right| \leq \frac{2 \epsilon_{j}}{\delta_{j} r_{j}}, \quad \forall(\xi, \lambda) \in \Pi_{j+1} .
\end{gathered}
$$

Moreover, we have

$$
\left|\lambda_{j+1}(\xi)-\lambda_{j}(\xi)\right| \leq \frac{2 \epsilon_{j}}{r_{j}}, \quad \forall(\xi, \lambda) \in \Pi_{j+1}
$$

The new perturbation $P_{j}$ satisfies that $\|P\|_{D_{j} \times \Pi_{j}} \leq \epsilon_{j}=\alpha r_{j} \rho_{j}^{\tau+n+1} E_{j}$.

In the following, we will check the assumptions in Lemma 2.4 to ensure that KAM step is valid for all $j \geq 0$.

Let $G_{j}=2 \epsilon_{j} / \delta_{j} r_{j}$. It follows that

$$
\frac{G_{j+1}}{G_{j}}=\frac{x_{j+1}^{n+\tau+1} e^{-x_{j+1}}}{x_{j}^{n+\tau+1} e^{-x_{j}}}
$$

where $x_{j}=K_{j} \rho_{j}$. By $E_{j+1}=c E_{j}^{3 / 2}$, if $E_{0}$ is sufficiently small, $E_{j}$ are all sufficiently small and so $x_{j}=K_{j} \rho_{j}$ are sufficiently large. Since the function $x^{n+\tau+1} e^{-x}$ decreases as $x>n+\tau+1$, we can choose a sufficiently small $E_{0}$ such that $G_{j+1} / G_{j} \leq 1 / 4$ and $G_{j} \leq 1 / 4$, for all $j \geq 0$. Moreover,

$$
\frac{\delta_{j+1}}{\delta_{j}}=\left(\frac{1}{2}\right)^{\tau+1}\left(\frac{x_{j}}{x_{j+1}}\right)^{\tau+1} \leq \frac{1}{4}
$$

Thus, the assumptions (2.25) and (2.30) hold.

\section{Convergence of the Iteration}

Now, we prove convergence of the KAM iteration. Let $\Pi_{*}=\bigcap_{j \geq 0} \Pi_{j}$ and $\Psi=\lim _{j \rightarrow \infty} \Psi_{j}$. In the same way as in $[4,13]$, we have the convergence $\Psi_{j}$ to $\Psi$ on $D(s / 2, r / 2) \times \Pi_{*}$, satisfying that

$$
\left\|W_{0}(\Psi-i d)\right\|_{D(s / 2, r / 2) \times \Pi_{*}} \leq c E_{0}
$$


Let $F_{j}=\sum_{i=0}^{j-1} f_{i}(\xi, \lambda)$. Now, we prove the convergence of $F_{j}$. Combining with the estimates for $f_{j}(\xi, \lambda)$, we have for all $(\xi, \lambda) \in \Pi_{j}$,

$$
\left|F_{j}(\xi, \lambda)\right| \leq \sum_{i=0}^{j-1} \frac{\delta_{i} G_{i}}{2} \leq \frac{\delta_{0}}{2} \sum_{i=0}^{j-1} G_{i} \leq \frac{2}{3} \delta_{0} G_{0} \leq \frac{2 \epsilon}{r}
$$

Similarly, it follows that for all $(\xi, \lambda) \in \Pi_{j}$,

$$
\left|F_{j \xi}(\xi, \lambda)\right|+\left|F_{j \lambda}(\xi, \lambda)\right| \leq \sum_{i=0}^{j-1} G_{i} \leq \frac{4}{3} G_{0}=\frac{16}{3} L x_{0}^{\tau+n+1} e^{-x_{0}}
$$

Then if $E_{0}$ is sufficiently small and so $x_{0}$ is sufficiently large, we have

$$
\left|F_{j \xi}(\xi, \lambda)\right|+\left|F_{j \lambda}(\xi, \lambda)\right| \leq \frac{1}{2}, \quad \forall(\xi, \lambda) \in \Pi_{j}
$$

the assumption (2.15) holds.

Let $F_{*}=\lim _{j \rightarrow \infty} F_{j}$, then for all $(\xi, \lambda) \in \Pi_{*}$, we have

$$
\begin{gathered}
\left|F_{*}(\xi, \lambda)\right| \leq \frac{2 \epsilon}{r}, \\
\left|F_{* \xi}(\xi, \lambda)\right|+\left|F_{* \lambda}(\xi, \lambda)\right| \leq \frac{1}{2} .
\end{gathered}
$$

This proves (2.7).

Let $\sigma_{*}=\sigma-(1 / 2) \sum_{j=0}^{\infty} \delta_{j}$. By (2.62), it follows that $\sigma_{*} \geq \sigma-(2 / 3) \delta_{0}$. If $E_{0}$ is sufficiently small such that $\delta_{0} \leq \sigma$, we have $\sigma_{*} \geq(1 / 3) \sigma$. Thus, $\Lambda_{\sigma_{*}} \subset \bigcap_{j \geq 0} \Lambda_{\sigma_{j}}$.

Similarly, we can prove the convergence of $\lambda_{j}(\xi)$ on $\Lambda_{\sigma_{*}}$. In fact, we can choose $E_{0}$ sufficiently small such that $G_{j} \leq 1 / 4$, for all $j \geq 0$. Then for $l \geq j$, it follows that

$$
\left|\lambda_{l}(\xi)-\lambda_{j}(\xi)\right| \leq \sum_{i=j}^{l-1} G_{i} \delta_{i} \leq \frac{4}{3} G_{j} \delta_{j} \leq \frac{\delta_{j}}{2} .
$$

Let $\lambda(\xi)=\lim _{l \rightarrow \infty} \lambda_{l}(\xi)$, then we have

$$
\left|\lambda(\xi)-\lambda_{j}(\xi)\right| \leq \frac{\delta_{j}}{2}
$$

This implies that $\Gamma_{\Omega}^{*}=\left\{(\xi, \lambda(\xi)) \mid \xi \in \Lambda_{\sigma_{*}}\right\} \subset \Pi_{j}$. So $\Gamma^{*}=\bigcup_{\Omega \in \mathcal{O}_{\alpha}} \Gamma_{\Omega}^{*} \subset \Pi_{*}=\bigcap_{j \geq 0} \Pi_{j}$. Moreover, for $(\xi, \lambda) \in \Gamma^{*}$, we have

$$
\bar{\omega}(\xi)+\lambda+F_{*}(\xi, \lambda)=\Omega
$$

Thus, the proof of Theorem 2.1 is complete. 


\section{Some Examples}

Example 3.1. We consider the following system:

$$
H=\bar{\omega}_{1} y_{1}+\frac{y_{1}^{4}}{4}+\bar{\omega}_{2} y_{2}+\frac{y_{2}^{4}}{4}+\epsilon\left(\sin \tilde{\omega}_{1} t+\sin \tilde{\omega}_{2} t\right)
$$

where

$$
N=\bar{\omega}_{1} y_{1}+\frac{y_{1}^{4}}{4}+\bar{\omega}_{2} y_{2}+\frac{y_{2}^{4}}{4}, \quad P=\epsilon\left(\sin \tilde{\omega}_{1} t+\sin \tilde{\omega}_{2} t\right)
$$

The frequency mapping

$$
\bar{\omega}(y)=\frac{\partial N}{\partial y}=\left(\bar{\omega}_{1}+y_{1}^{3}, \bar{\omega}_{2}+y_{2}^{3}\right)
$$

at $y=0$ does not satisfy the Kolmogorov's nondegeneracy condition. But

$$
\operatorname{Rank}\left\{\bar{\omega}(y), \frac{\partial \bar{\omega}(y)}{\partial y}\right\}=\operatorname{Rank}\left\{\begin{array}{ccc}
\bar{\omega}_{1}+y_{1}^{3} & 3 y_{1}^{2} & 0 \\
\bar{\omega}_{2}+y_{2}^{3} & 0 & 3 y_{2}^{2}
\end{array}\right\}=2
$$

So according to our theorem, if $\epsilon$ is sufficiently small, $\omega=(\bar{\omega}, \tilde{\omega})$ satisfies the Diophantine condition and $\operatorname{deg}(\bar{\omega}(y), D, \bar{\omega}) \neq 0$, the perturbed system still has an invariant torus with $\omega=$ $(\bar{\omega}, \tilde{\omega})$ as its frequency.

Example 3.2. We consider the following quasiperiodic mapping $A$ :

$$
\begin{gathered}
x_{1}=x+\omega+\beta(y)+f(x, y), \\
y_{1}=y+g(x, y),
\end{gathered}
$$

where $f$ and $g$ are quasiperiodic in $x$ with frequencies $\mu_{1}, \ldots, \mu_{m}$, real analytic in $x$ and $y$, the variable $y$ ranges in a neighborhood of the origin of real line $\mathbb{R}, \omega$ is a positive constant. Suppose that the mapping $A$ is reversible with respect to the involution $R:(x, y) \rightarrow(-x, y)$, that is $A \circ R \circ A=R$.

When $d \beta(y) / d y \neq 0, \omega$ satisfies certain Diophantine condition and $f, g$ are sufficiently small, the existence of invariant curve with $\omega$ as its frequency has been proved in $[19,20]$. The condition $d \beta(y) / d y \neq 0$ is called twist condition. The natural question is when the condition $d \beta(y) / d y \neq 0$ is not satisfied, that is, there is some $y^{*}$ such that $d \beta\left(y^{*}\right) / d y=0$, whether there exists invariant curve for mapping (3.5), whether its frequency can persist without any drift. By the method of introducing an external parameter as in our paper, we can prove that the mapping (3.5) still has an invariant curve with $\omega$ as its frequency, when $\beta(y)=y^{2 n+1}$. For detailed proofs, we refer to [21].

Remark 3.3. When $\beta(y)=y^{2 n}$, we can only prove the existence of invariant curve for the mapping (3.5), but its frequency has some drifts. 


\section{Acknowledgments}

The work was supported by the National Natural Science Foundation of China (no. 10826035), (no. 11001048) and the Specialized Research Fund for the Doctoral Program of Higher Education for New Teachers (no. 200802861043). It was also supported by the Science Research Foundation of Nanjing University of Information Science and Technology (no. 20070049).

\section{References}

[1] V. I. Arnold, "Proof of a theorem of A. N. Kolmogorov on the preservation of conditionally periodic motions under a small perturbation of the Hamiltonian," Uspekhi Matematicheskikh Nauk, vol. 18, no. 5 (113), pp. 13-40, 1963.

[2] L. H. Eliasson, "Perturbations of stable invariant tori for Hamiltonian systems," Annali della Scuola Normale Superiore di Pisa, vol. 15, no. 1, pp. 115-147, 1988.

[3] A. N. Kolmogorov, "On conservation of conditionally periodic motions for a small change in Hamilton's function," Doklady Akademii Nauk SSSR, vol. 98, pp. 527-530, 1954.

[4] J. Pöschel, "A lecture on the classical KAM theorem," in Smooth Ergodic Theory and Its Applications (Seattle, WA, 1999), vol. 69 of Proc. Sympos. Pure Math., pp. 707-732, American Mathematical Society, Providence, RI, USA, 2001.

[5] C. Q. Cheng and Y. S. Sun, "Existence of KAM tori in degenerate Hamiltonian systems," Journal of Differential Equations, vol. 114, no. 1, pp. 288-335, 1994.

[6] H. Rüssmann, "Nondegeneracy in the perturbation theory of integrable dynamical systems," in Stochastics, Algebra and Analysis in Classical and Quantum Dynamics (Marseille, 1988), vol. 59 of Math. Appl., pp. 211-223, Kluwer Acad. Publ., Dordrecht, The Netherlands, 1990.

[7] H. Rüssmann, "Invariant tori in non-degenerate nearly integrable Hamiltonian systems," Regular $\mathcal{E}$ Chaotic Dynamics, vol. 6, no. 2, pp. 119-204, 2001.

[8] M. B. Sevryuk, "KAM-stable Hamiltonians," Journal of Dynamical and Control Systems, vol. 1, no. 3, pp. 351-366, 1995.

[9] J. Xu, J. You, and Q. Qiu, "Invariant tori for nearly integrable Hamiltonian systems with degeneracy," Mathematische Zeitschrift, vol. 226, no. 3, pp. 375-387, 1997.

[10] S.-N. Chow, Y. Li, and Y. Yi, "Persistence of invariant tori on submanifolds in Hamiltonian systems," Journal of Nonlinear Science, vol. 12, no. 6, pp. 585-617, 2002.

[11] M. B. Sevryuk, "Partial preservation of frequencies in KAM theory," Nonlinearity, vol. 19, no. 5, pp. 1099-1140, 2006.

[12] J. Xu and J. You, "Persistence of the non-twist torus in nearly integrable Hamiltonian systems," Proceedings of the American Mathematical Society, vol. 138, no. 7, pp. 2385-2395, 2010.

[13] B. Liu, S. Shi, and G. Wang, "KAM-type theorem for nearly integrable Hamiltonian with a quasiperiodic perturbation," Northeastern Mathematical Journal, vol. 19, no. 3, pp. 273-282, 2003.

[14] H. Whitney, "Analytic extensions of differentiable functions defined in closed sets," Transactions of the American Mathematical Society, vol. 36, no. 1, pp. 63-89, 1934.

[15] J. Bonet, R. W. Braun, R. Meise, and B. A. Taylor, "Whitney's extension theorem for nonquasianalytic classes of ultradifferentiable functions," Studia Mathematica, vol. 99, no. 2, pp. 155-184, 1991.

[16] J. Xu and J. You, "Gevrey-smoothness of invariant tori for analytic nearly integrable Hamiltonian systems under Rüssmann's non-degeneracy condition," Journal of Differential Equations, vol. 235, no. 2, pp. 609-622, 2007.

[17] D. Zhang and J. Xu, "Gevrey-smoothness of elliptic lower-dimensional invariant tori in Hamiltonian systems under Rüssmann's non-degeneracy condition," Journal of Mathematical Analysis and Applications, vol. 323, no. 1, pp. 293-312, 2006.

[18] D. Zhang and J. Xu, "On elliptic lower dimensional tori for Gevrey-smooth Hamiltonian systems under Rüssmann's non-degeneracy condition," Discrete and Continuous Dynamical Systems. Series A, vol. 16, no. 3, pp. 635-655, 2006.

[19] B. Liu, "Invariant curves of quasi-periodic reversible mappings," Nonlinearity, vol. 18, no. 2, pp. 685701, 2005. 
[20] V. Zharnitsky, "Invariant curve theorem for quasiperiodic twist mappings and stability of motion in the Fermi-Ulam problem," Nonlinearity, vol. 13, no. 4, pp. 1123-1136, 2000.

[21] D. Zhang and J. Xu, “On invariant curves of analytic reversible mappings with degeneracy," Far East Journal of Applied Mathematics, vol. 37, no. 3, pp. 315-334, 2009. 